\title{
SIMULATION STUDIES OF SPACE-CHARGE-DOMINATED BEAMS IN SPALLATION NEUTRON SOURCES *
}

\author{
J. A. Holmes, J. D. Galambos, D. Jeon, V. V. Danilov, D. K. Olsen, ORNL, Oak Ridge, TN, \\ M. Blaskiewicz, BNL, Upton, NY, S. Y. Lee, Indiana University, Bloomington, IN
}

\begin{abstract}
Uncontrolled beam losses due to space-charge-induced halo generation are a concern in the accumulator rings of spallation neutron sources, such as SNS and ESS. Such rings are characterized by high beam intensities and low uncontrolled beam loss requirements. It is therefore important to investigate the dynamics of space charge in high intensity rings. We have done this extensively by using a particle tracking approach with a self-consistent particle-in-cell code. We have found that the inclusion of space charge forces is essential in simulating not only the dynamics of halo generation, but also the formation of the observed beam profile shapes under typical conditions. Our results extend the understanding of fundamental space charge physics, which has been developed for linear accelerators, to rings.
\end{abstract}

\section{INTRODUCTION}

Accumulator rings for spallation neutron sources represent a new regime for the study of space charge dynamics. In comparison with linear accelerators, for which much understanding of space charge dynamics has been gained [1], space charge forces are modest in rings, with tune shifts of only a few percent of the bare tunes. However, beams remain in accumulator rings much longer than in linacs, and have significant time to become destabilized. Furthermore, the lattice periodicities and dispersion phenomena peculiar to rings couple to space charge effects to enrich and complicate the dynamic picture.

The motivation to study space charge dynamics in high intensity rings is related to their extremely low beam loss requirements. For example, to facilitate hands-on maintenance in the SNS accumulator ring, uncontrolled losses from the $1 \mathrm{GeV}$ proton beam are limited to < 1 $n A / m$, or about 1 part in $10^{4}$ [2]. Consequently, uncontrolled beam losses due to space-charge-induced halo generation are an important concern.

We have extensively examined the effects of space charge in high intensity rings using a particle tracking approach with a self-consistent particle-in-cell code, ORBIT [3]. Section 2 briefly presents the computational model used in our studies. A more complete description can be found in Ref. [4]. Section 3 presents a detailed comparison of calculated and measured beam profiles for the PSR accumulator ring at LANL [5]. The calculations match as closely as possible the actual injection scheme and beam accumulation process occurring in PSR. These results serve not only to benchmark ORBIT, but also to emphasize the necessity of including space charge forces in the calculations. Section 4 describes some new results on the role of space-charge-driven lattice resonances in creating rms mismatch in initially matched beams [6]. This mismatch can then drive the parametric resonance in the usual way, resulting in large halo formation [7]. Section 5 presents the results of injection studies for the SNS ring including a survey of halo formation as a function of vertical tune and a comparison of results for comparable FODO and doublet lattices. Finally, our conclusions are presented in Sect. 6.

\section{COMPUTATIONAL MODEL}

High intensity rings are characterized by the separation of longitudinal and transverse scales. In SNS, for example, the longitudinal bunch length is on the order of $100 \mathrm{~m}$, compared with transverse beam dimensions of a few $\mathrm{cm}$; and the longitudinal tune is about $10^{-3}$, compared with transverse tunes of about 5.8. For this reason it is possible, with good approximation, to separate the longitudinal and transverse dynamics in high intensity rings and, for the study of space charge effects, to consider the transverse dynamics. In ORBIT, the particle tracking is carried out in six dimensional phase space and, although transverse canonical coordinates couple strongly into the longitudinal equations, the only coupling of longitudinal dynamics into the transverse equations is through the momentum deviation $\delta \equiv \Delta p / p_{0}$ and the modulation of the space charge density along the bunch. For the remainder of this paper we will concentrate on space charge in transverse phase space.

The model adopted in the ORBIT code is a particletracking approach. The essence of the transverse phase space treatment is the following dynamic equation for the macroparticle coordinate $u=x$ or $y$ :

$$
u^{\prime \prime}=F_{u}^{l i n}+F_{u}^{s c}+F_{u}^{n l},
$$

where prime denotes differentiation with respect to the longitudinal coordinate $s, F_{u}^{\text {lin }}$ are the linear magnet forces, $F_{u}^{s c}$ are the space charge forces, and $F_{u}^{n l}$ are the nonlinear magnet forces. The linear focusing forces and nonlinear magnet forces are dependent on the lattice and are evaluated independently for each macroparticle using first and second order transport matrices, respectively, generated by either MAD [8] or DIMAD [9]. The space 
charge forces are collective, involving the interacton of all the macroparticles, and are evaluated self-consistently:

$$
F_{u}^{s c}=P \sum_{i=1}^{N} \frac{u-u_{i}}{\left(x-x_{i}\right)^{2}+\left(y-y_{i}\right)^{2}},
$$

where the summation is over the number of macroparticles, $N$, and $P$ is the generalized perveance of the beam [10].

The integration scheme is chosen to be explicit second order symplectic, with a transport matrix representation of all external magnet forces and the inclusion of space charge forces as kicks. Our treatment of space charge uses a particle-in-cell (PIC) model [11] with fast Fourier transforms (FFTs) to evaluate the forces. Further details of the algorithms are given in Ref. [4]. The inclusion of nonlinear magnet forces in the model is recent, and we do not utilize this feature in the present results. Consequently, the space charge potential provides the only nonlinearity in these calculations.

The study of space charge using this numerical model involves a number of parameters: $N$, the number of macroparticles; $N_{F F T}$, the spatial resolution (grid parameter for the FFT algorithm); and $N_{a z}$, the number of azimuthal integration steps. Convergence studies have shown that the accuracy of the solution depends both on having a sufficient number of macroparticles, $N$, and enough FFT gridpoints, $N_{F F T} \times N_{F F T}$, but that the numerical stability of the integration scheme depends only on having a sufficiently fine azimuthal grid, as determined by $N_{a z}$. The results presented here were obtained using $N=(2-10) \times 10^{4}$ macroparticles and $N_{F F T} \times N_{F F T}=$ $64 \times 64$ FFT grid points. For numerical stability we used $N_{a z}=480$ integration points, which corresponds to about 20 points per FODO cell or step sizes of about $1 / 2$ meter. Such calculations typically require about 10 hours on a $500 \mathrm{MHz}$ Dec Alpha workstation.

\section{BENCHMARK WITH PSR}

As a benchmark of the ORBIT code, we now present comparisons of simulated and experimentally measured transverse beam profiles at the end of injection in the Los Alamos Neutron Science Center, LANSCE, Proton Storage Ring, PSR. Experimental beam profiles were generated using $\mathrm{H}^{-}$foil injection from an $800 \mathrm{MeV}$ linac. Injection was carried out for a duration of $825 s$, or 2305 turns, for two painting schemes and three beam intensities. Subsequently, the beam was extracted in a single turn and transported to a wire scanner beam profile diagnostic in the extraction beam line.

In the benchmark calculations, care was taken to match the full PSR lattice parameters, injection scenarios, and beam intensities [5]. Because the absolute transverse location of the wire scanner was not precisely known, the comparison was carried out by matching the centers of the calculated and experimental distributions. The shift required to align these proved to be independent of beam intensity. Also, because the calculated and measured distributions were obtained in different arbitrary units, the vertical scales of the distributions were adjusted so that the calculated height matched that from the wire scanner data at the center of the distribution. We emphasize that the width and shape of the calculated distribution were not adjusted. Comparisons were made for two injection scenarios, both with and without a vertical closed orbit bump at the foil. The PSR does not have bump magnets to paint in the horizontal direction, but a horizontal spread is provided through the injected beam energy distribution since there is a dispersion of $1.4 \mathrm{~m}$ at the foil location.

The overall results reveal very good agreement between the measured and calculated transverse profiles. For both injection scenarios, there is a noticeable broadening of the vertical distribution with increasing beam intensity in both the experimental measurements and calculated profiles. The horizontal profiles are much less sensitive to increasing beam intensity, both for the experimental and calculated results.

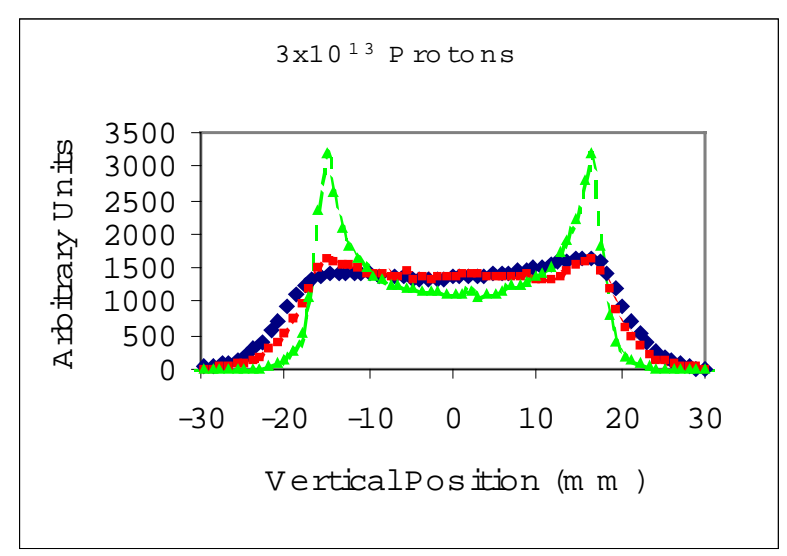

Figure 1. The effect of the space charge force on the calculated beam profiles, for the highest intensity case with no closed orbit bumps during injection. Blue curve experiment; red curve - calculation with space charge; green curve - calculation without space charge.

Furthermore, this good agreement is very dependent on including the space charge force in the calculations. This is demonstrated emphatically in Fig. 1, which shows the experimental and calculated vertical beam profiles at full intensity, calculated both with and without the space charge force. For this case the injection is offset vertically with no bumps. This is reflected by the "horns" on the profile calculated without the space charge force, which occur at the location of injection with peak width corresponding to the linac beam size. In both the experiment and the calculation with space charge, space charge forces spread these "horns", causing the distribution to fill in and to broaden. The transverse space charge model predicts most of the observed beam 
broadening. This comparison provides a benchmark that the space charge calculations are correct, particularly considering that the intensity of the PSR beam at high currents is comparable to that in proposed spallation neutron sources.

\section{LATTICE RESONANCES AND HALO FORMATION}

It is well established, both in linacs and rings, that a primary cause of space-charge-induced halo formation is rms beam mismatch [1,7]. This mismatch leads to envelope oscillations at approximately twice the coherent tune which, driven by space charge forces, manifest as the parametric resonance. Beam particles with large action in the phase plane of the resonance can diffuse across the separatrix and become halo particles. Although it is necessary to have well-matched beams to avoid halo formation, we now show that this may not be sufficient. Depending on the operating point in tune space, space charge forces can drive resonances that lead to beam mismatch, even for initially rms-matched beams. The presentation here is brief, and greater details can be found in Ref. [6, Jeon et al].

In order to illustrate these phenomena clearly, we calculate three cases in the fourfold symmetric SNS ring lattice using initially matched beams with K-V distributions. For these cases the initial transverse rms beam emittances are chosen to be $120 \pi \mathrm{mm}$ - $\mathrm{mrad}$, the proton beam energy is $1 \mathrm{GeV}$, the beam intensity is set so that the initial tune shift is about 0.08 giving a tune depression of $1.4 \%$, and the initial horizontal bare tune is $v_{x}=5.82$. The three cases are identical except for varying the choice of vertical bare tune $v_{y}$, which is taken to be $v_{y}=5.67,5.82$, and 5.77.

Before proceeding, we note that an ideal K-V distribution supports only even terms in the space charge potential. However, in general, all terms will be present if there are asymmetric nonuniformities in the beam distribution. Because the numerically-generated $\mathrm{K}-\mathrm{V}$ beams used in these calculations are created with random numbers, the resulting distributions contain small nonuniformities. If the operating point in tune space is sufficiently close to certain resonances, it is possible for the space charge nonuniformities to excite these resonances and destabilize the beam. As shown below, this will apply even for the case of an rms matched beam.

Considering first the case, $v_{y}=5.67$, the operating point is such that no lattice resonances are excited. In a calculation tracking the rms matched beam for 1250 turns, the transverse rms emittances are observed to remain constant, envelope fluctuations remain small, and the beam phase space plots show no halo. To observe halo generation for this operating point, it is necessary to mismatch the initial envelope radii by more than about $5 \%$, beyond which the extent and population of the halo increases with increasing mismatch.
We next consider the case of $v_{x}=v_{y}=5.82$. This point in tune space lies atop the difference resonance $v_{x}-v_{y}=0$. Difference resonances $v_{x}-v_{y}=n$ are not usually regarded as dangerous to the operation of accelerators because of the conservation of $J_{x}+J_{y}$, where $J_{x(y)}$ is the $x(y)$ action. However, it has been shown [12] using self-consistent Vlasov-Poisson equations that the space charge potential can support an odd mode contribution $\propto x y$ capable of exciting the difference resonance. Given the presence of such a driving term, the difference resonance, $v_{x}-v_{y}=0$, facilitates halo generation in two ways: (1) by increasing the strength of the $2: 1$ parametric resonance through the coupling of the horizontal and vertical envelope oscillations; and (2) by helping particles, through coupling, to cross the separatrices of the 2:1 parametric resonance.

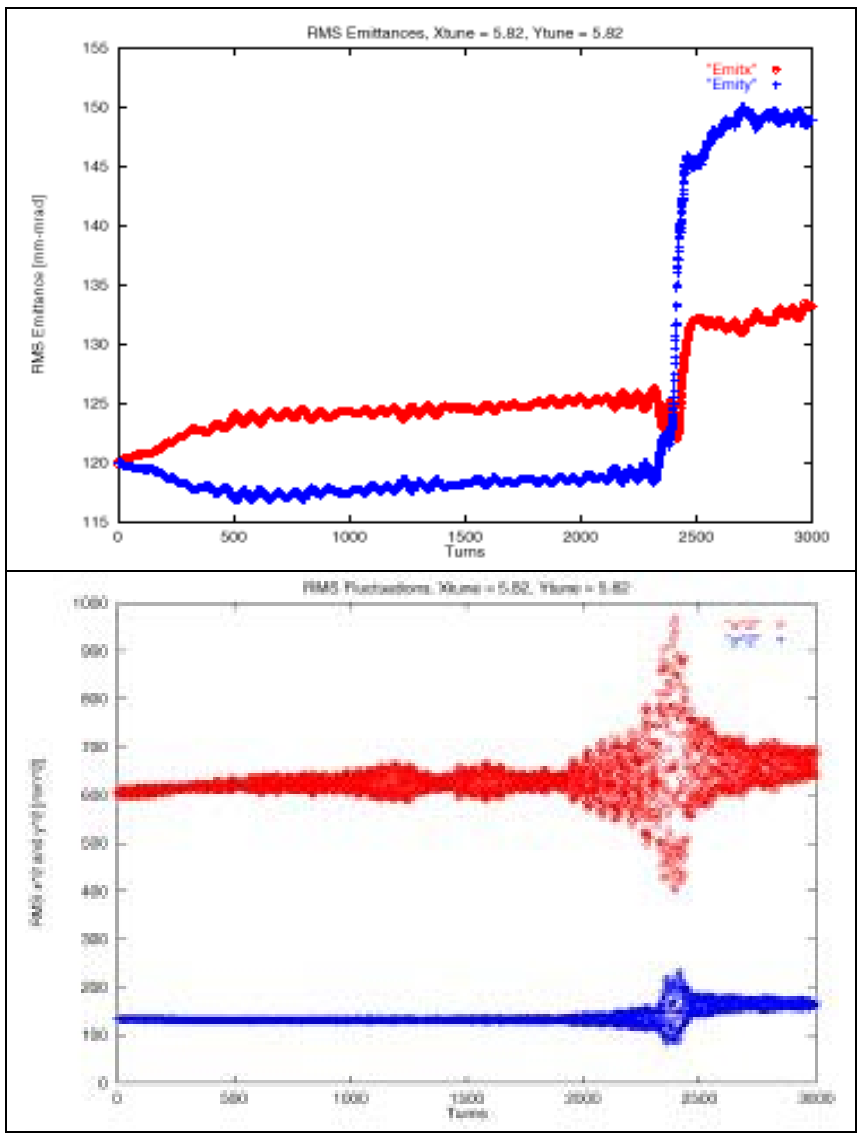

Figure 2. Evolution of rms emittances and second moments $\left\langle\Delta x^{2}>\right.$ and $\left\langle\Delta y^{2}>\right.$ for $v_{x}=v_{y}=5.82$.

Figure 2 shows the evolution of the transverse rms emittances and envelope fluctuations for the case of a well-matched beam at $v_{x}=v_{y}=5.82$. The small initial oscillations in the second moments reflect the quality of the match. Even though the odd mode contribution $\propto x y$ 
is small in the potential of the numerical $\mathrm{K}-\mathrm{V}$ distribution, this term excites the difference resonance. Evidence of this is clear in the anticorrelation of the horizontal and vertical emittances in Fig. 2. This leads to rms mismatch, as shown by the increasing oscillation amplitudes of $<\Delta x^{2}>$, and ultimately to the strong excitation of the $2: 1$ parametric resonance. Little halo formation is observed for this case for over 2000 turns, but ultimately substantial halo formation accompanies a large emittance increase at around 2300 turns. The strength of this process is due to the difference resonance enhancing the parametric resonance by coupling the horizontal and vertical envelope oscillations and by helping particles to cross the separatrix through $x-y$ coupling.

Let us now consider the case of $v_{x}=5.82$ and $v_{y}=$ 5.77. In this case the fourth order resonance $4 v_{y}=23$ is excited by the fourth order even mode term, $a_{0} x^{4}+a_{2} x^{2} y^{2}+a_{4} y^{4}$, in the space charge potential. Again, for an initially well-matched beam, this induces a mismatch that leads to significant halo formation. Unlike odd mode terms in the space charge potential, which are skew terms in the Hamiltonian that are usually small in lattices with midplane symmetry, even mode terms can be significant. Consequently, when the space charge potential is the driving force in such lattices, the $4 v_{y}=n$ resonance associated with the fourth order even mode potential term is significantly more excited than the $3 v_{y}=$ $n$ resonance associated with the third order odd mode term, $a_{1} x^{2} y+a_{3} y^{3}[12]$.

When the coherent tune is near a lattice resonance [13] associated with an even mode space charge potential, the excited resonance can generate a mismatch, even though the initial beam is well matched. This mismatch can drive the 2:1 parametric resonance and expedite halo formation. Figure 3 illustrates this process for the case with $v_{x}=5.82$ and $v_{y}=5.77$. The moderate $y \mathrm{rms}$ emittance increase around 200 turns is due to the excitation of the fourth order $4 v_{y}=23$ resonance. This can be seen in the upper right plot, which is a snapshot of the particle distribution in $y$ phase space taken at 250 turns. An rms beam mismatch is generated by this resonance as can be seen in the plot of the beam moments at the lower left. This mismatch leads to the excitation of the 2:1 parametric resonance and to the subsequent large increase in the $y$ emittance. Because the tune difference is only $v_{x}-v_{y}=$ 0.05 , the difference resonance also contributes to the strength of the process. This can be seen in the anticorrelation of the $x$ and $y$ emittances, an effect that is due to coupling. The $y$ phase space plot at lower right superposes the beam distribtuions at 1000 turns and 1250 turns, and shows the 1:2 parametric resonance structure and halo. Clearly, it is advantageous to avoid nonlinear resonances associated with even mode space charge potentials when choosing an operating point for intense beam circular accelerators. This is especially true when the horizontal and vertical tunes are close.

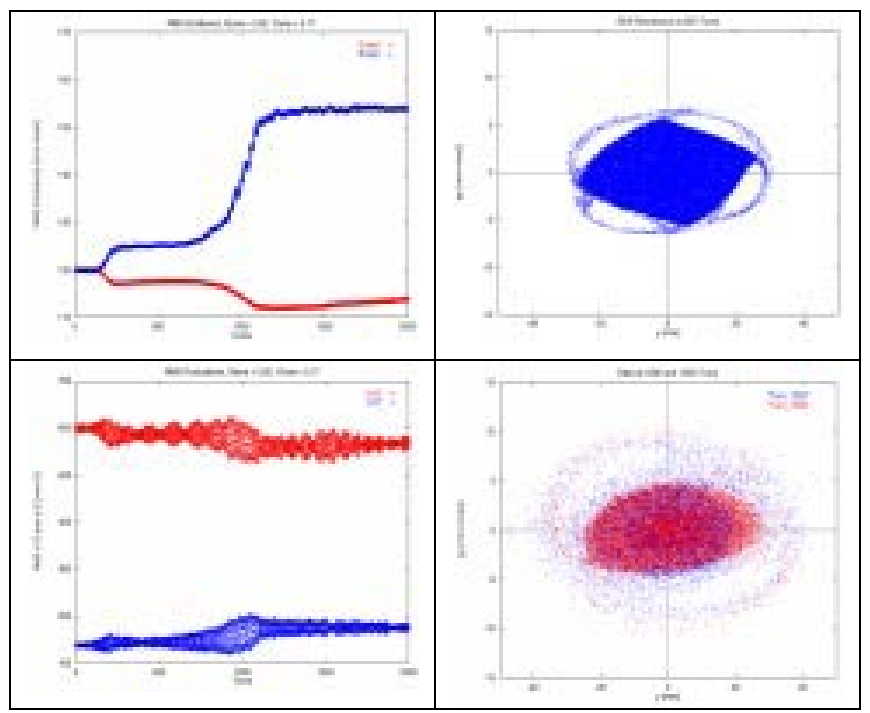

Figure 3. Evolution of rms emittances and second moments $\left\langle\Delta x^{2}\right\rangle$ and $\left\langle\Delta y^{2}\right\rangle$ for $v_{x}=5.82, v_{y}=5.77$.

Phase space plots in vertical plane for 250 turns and superposition of 1000 and 1250 turns.

The main conclusion of this section is that, depending on the operating point in tune space, space charge forces can excite resonances that lead to beam mismatch. This mismatch can then excite the parametric resonance, thus leading to substantial halo formation, even for initially matched beams. Furthermore, if the $x$ and $y$ tunes are close together the resulting coupling can contribute to the destabilization of the parametric resonance and the resulting halo formation.

\section{INJECTION STUDIES}

We have carried out a number of injection studies for the SNS ring, including optimization of the injection scheme for minimum halo formation [14]. The most recent injection studies include a survey of vertical tune space for fixed horizontal tune, $v_{x}=5.82$, and a comparison of doublet and FODO lattices having the same global parameters, including length, beam size, and bare tunes. These studies track the entire injection process, including such features as incident linac beam distribution, foil stripping model, and bumping schemes, for 1158 turns. More details of the injection model are presented in Reference [14].

Figure 4 shows the results of the vertical tune survey by plotting the percentage of particles with $x$ or $y$ emittance exceeding $180 \pi \mathrm{mm}$-mrad as a function of bare tune at the end of injection. The resolution of this tune scan is coarse, with cases separated by $\Delta v_{y}=0.05$ in the range from $4<v_{y} \leq 6$, but it is clear that halo formation is sensitive to the operating point in tune space. It is 
interesting that, although bare tunes of exactly 5 or 6 give low halo formation, the worst operating points flank these integral values. The divergence of the results as $v_{y}$ approaches 4 is due to the structure resonance with the fourfold symmetric SNS lattice. We also note that there are a number of operating points at which none of the particles exceed $180 \pi \mathrm{mm}$-mrad, indicating that it may be possible to avoid producing halo through proper choice of operating point.

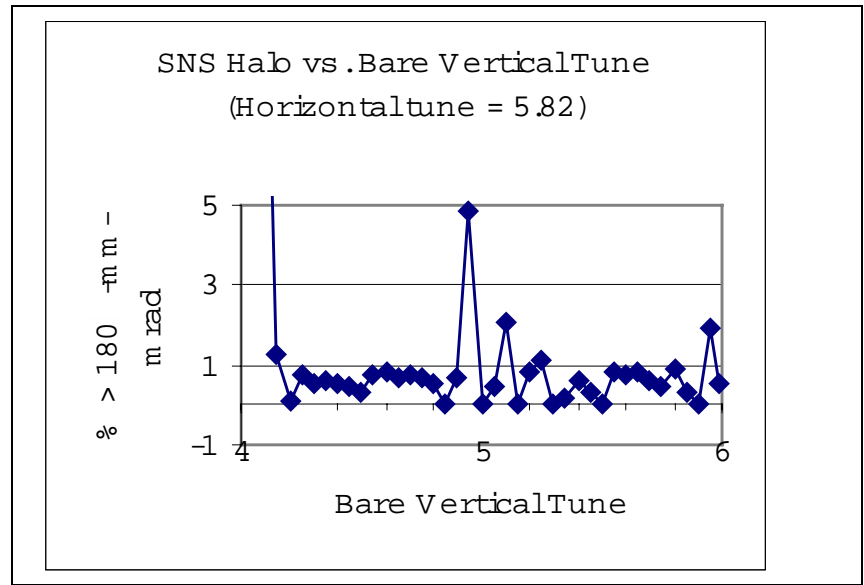

Figure 4. Halo production as a function of vertical tune for injection into SNS lattice with $v_{x}=5.82$.

Finally, calculations were carried out comparing halo formation for doublet and FODO lattices having the same global parameters. For both lattices, the results are sensitive to the tune values, but in some cases the doublet lattice produces less halo and in other cases the FODO lattice produces less.

\section{CONCLUSIONS}

We have demonstrated by benchmark comparison with experiment that the inclusion of space charge forces is a necessary component in any beam-dynamic model of high intensity rings. We have also shown that nonlinear space charge forces can excite various lattice resonances that lead to rms beam mismatch. This can trigger a two-stage process in which the mismatched beam in resonance with the lattice excites the parametric resonance, resulting in substantial halo formation. These results, along with realistic injection studies surveying the tune dependence of halo formation, emphasize the sensitivity of beam stability to the operating point in tune space. Comparison of halo formation in FODO and doublet lattices with the same global parameters shows that results are sensitive to the tune, but reveal no performance bias for one or the other. Future work will involve the study of space charge dynamics in the presence of nonlinear magnetic forces, such as errors, fringe fields, and higher multipoles, which have recently been incorporated into the ORBIT Code.
* Research on the Spallation Neutron Source is sponsored by the Division of Materials Science, U.S. Department of Energy, under contract number DE-AC05-96OR22464 with Lockheed Martin Energy Research Corporation for Oak Ridge National Laboratory.

\section{REFERENCES}

[1] P.M. Lapostolle, IEEE Trans. Nucl. Sci. NS-18, 1101 (1971); F.J. Sacherer, ibid. NS-18, 1105 (1971); J.D. Lawson P.M. Lapostolle, and R.L. Gluckstern, Part. Accel. 5, 61 (1973); E.P. Lee and R.K. Cooper, ibid. 7, 83 (1976); C. Chen and R.C. Davidson, Phys. Rev. E 49, 5679 (1994); Phys. Rev. Lett. 72, 2195 (1994); S.Y. Lee and A. Riabko, Phys. Rev. E 51, 1609 (1995); A. Riabko, et al, Phys. Rev. E 51, 3529 (1995); R.A. Jameson, Proceedings of the 1993 Particle Accelerator Conference (IEEE, Piscataway, NJ, 1993), p.3926 (unpublished); J.S. O'Connell, T.P. Wangler, R.S. Mills, and K.R. Crandall, Proceedings of the 1993 Particle Accelerator Conference, (IEEE, Piscataway, 1993), p.3657; J.M. Lagniel, Nucl. Instr. Meth. A345, 46 (1994); A345, 405 (1994); I. Hofmann, L.J. Laslett, L. Smith, and I. Haber, Part. Accel. 13, 145 (1983); Struckmeier and M. Reiser, Part. Accel. 14, 227 (1983); R. L. Gluckstern, Phys. Rev. Lett. 73 (1994) $1247 .$.

[2] National Spallation Neutron Source Conceptual Design Report,Volumes 1 and 2, NSNS/CDR-2/V1,2, (May, 1997); at http://www.ornl.gov/ nsns/CDRDocuments/CDR.html

[3] Galambos, J.D., Holmes, J.A., and Olsen, D.K., ORBIT User Manual, Version 1.01, SNS/ORNL/AP TECHNICAL NOTE Number 011, (March, 1999).

[4] J.A. Holmes et al, Proceedings of International Computational Accelerator Physics Conference, (Monterey, CA, September 1998); F. Jones, Users' Guide to ACCSIM, TRIUMF Design Note, TRIDN-90-17 (1990).

[5] J.D. Galambos et al, submitted to PRSTAB, (1999).

[6] S. Machida and Y. Shoji, in Space Charge Dominated Beams and Applications of High Brightness Beams, AIP Conf. Proc. 377, ed. S.Y. Lee, 161 (1995); D. Jeon et al, submitted to Phys. Rev. Lett., (1999).

[7] S. Machida, in Workshop on Space Charge Physics in High Intensity Hadron Rings, AIP Conf. Proc. 488, ed. A.U. Luccio, W.T. Weng, 73 (1998); J.A. Holmes et al, Proceedings of the European Particle Accelerator Conference, paper THP24C, (Stockholm, June 1998).

[8] H. Grote and F. Christoph Iselin, The Mad Program, Version 8.19, User's Reference Manual, CERN/SL/90-13 (Geneva, 1996).

[9] R.V. Servranckx, K.L. Brown, L. Schachinger, and D. Douglas, User's Guide to the Program DIMAD, SLAC Report 285 UC-28 (A), (May, 1985).

[10] Thomas P. Wangler, in Space Charge Dominated Beams and Applications of High Brightness Beams, AIP Conf. Proc. 377, ed. S.Y. Lee, 3 (1995).

[11]R.W. Hockney and J.W. Eastwood, Computer Simulation Using Particles, Adam Hilger, IOP Publishing Ltd. (New York: 1988); C.K. Birdsall and A.B. Langdon, Plasma Physics via Computer Simulation, McGraw-Hill Book Company (New York: 1985).

[12]I. Hofmann, Phys. Rev. E 57, 4713 (1998).

[13]R. Baartman, Proceedings of Workshop on Space Charge Physics in High Intensity Hadron Rings (Shelter Island, NY, 1998), p.56.

[14]J.D. Galambos, J.A. Holmes, and D.K. Olsen, Proceedings of the Particle Accelerator Conference, ,(Vancouver, 1997). 